\title{
Application of Team Resource Management in Enhancing the Chemotherapy Administration Safety \\ in a Medical center of Taiwan
}

\section{Fang - Chun Lin}

Department of Nursing, Shin Kong Wu Ho-Su Memorial Hospital, Taipei,

\author{
Taiwan
}




\section{KEY WORD}

- team resources management

- chemotherapy administration

- chemotherapy administration incidence rate 


\section{BACKGROUND}

The chemotherapy administration incidence rates increased from $0.008 \%$ to $0.04 \%$ during2014 to 2015.This project explored the effects of application of team resource management intervention enhance the patient safety of chemotherapy administration in a medical center of Taiwan. 


\section{OBJECTIVE}

The purpose of this project explored the effects of application of team resource management intervention enhance the patient safety of chemotherapy administration in a medical center of Taiwan. 


\section{METHODS}

Applied the root cause analysis on the collected data from 2015 to 2018.The total numbers of chemotherapy administration were 49,894, with a total of 11 incident events. Extravasation accounted for $90.9 \%$, with swelling (38.4\%), pain (30.8\%), red (23.1\%) symptoms, 5FU accounted for the most; administration error accounted for $9.1 \%$. 


\section{METHODS}

The problems identified were incorrect of port-A catheter fixation and angle needle size selection, insufficient awareness of chemotherapy extravasation, complex chemotherapy orders without standard prescription, calculation error in the flow rate,absence of double checking, lack of pharmacy information and further training. 


\section{METHODS}

We then develop a series improvement strategies included by medical resource integration, develop of port-A catheter care DVD, chemotherapy extravasation prevention handbook, integrated chemotherapy order package by information system, implementation of barcode administration system, and educational training programs for safety administration and standardizing . 


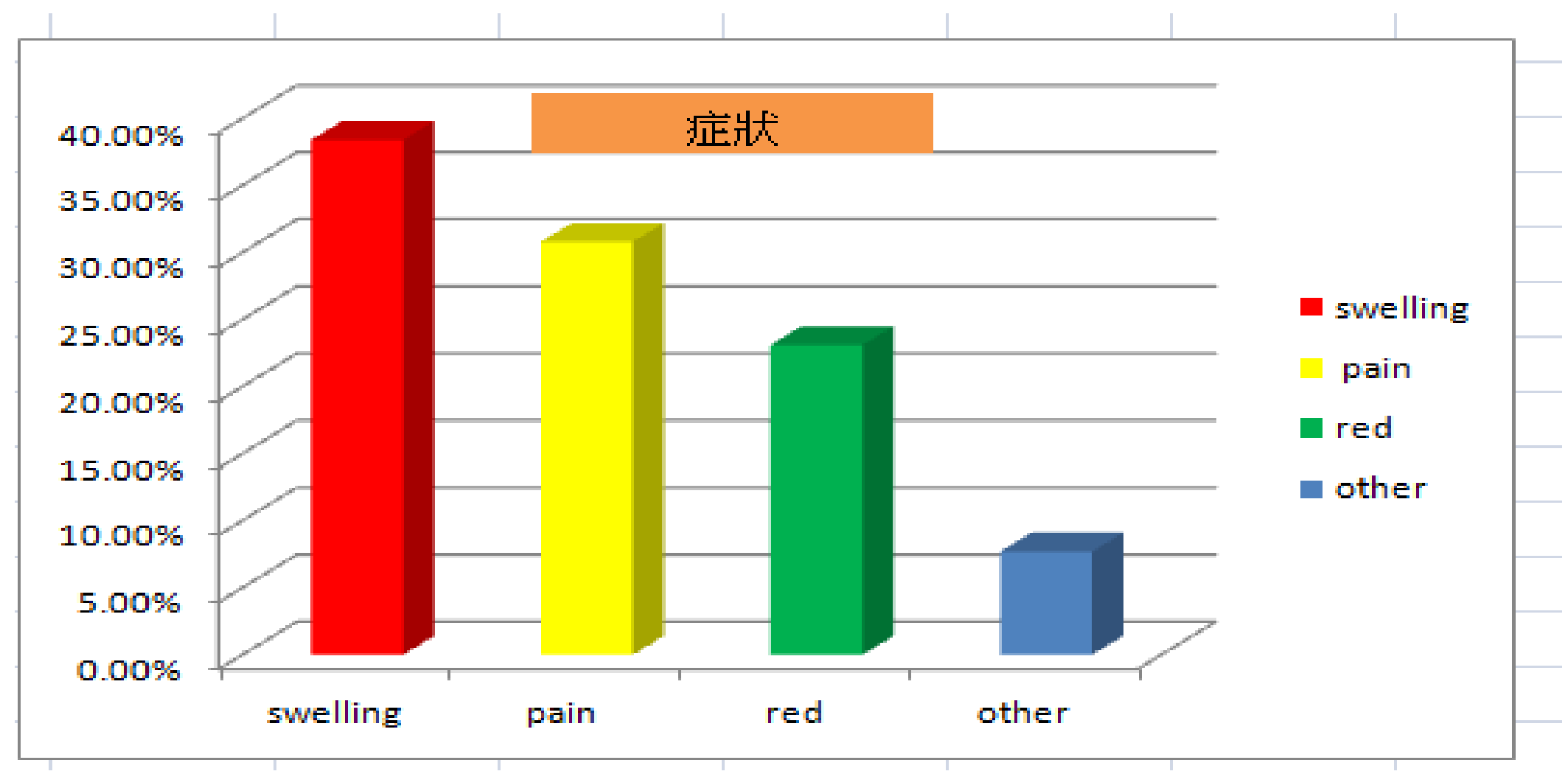



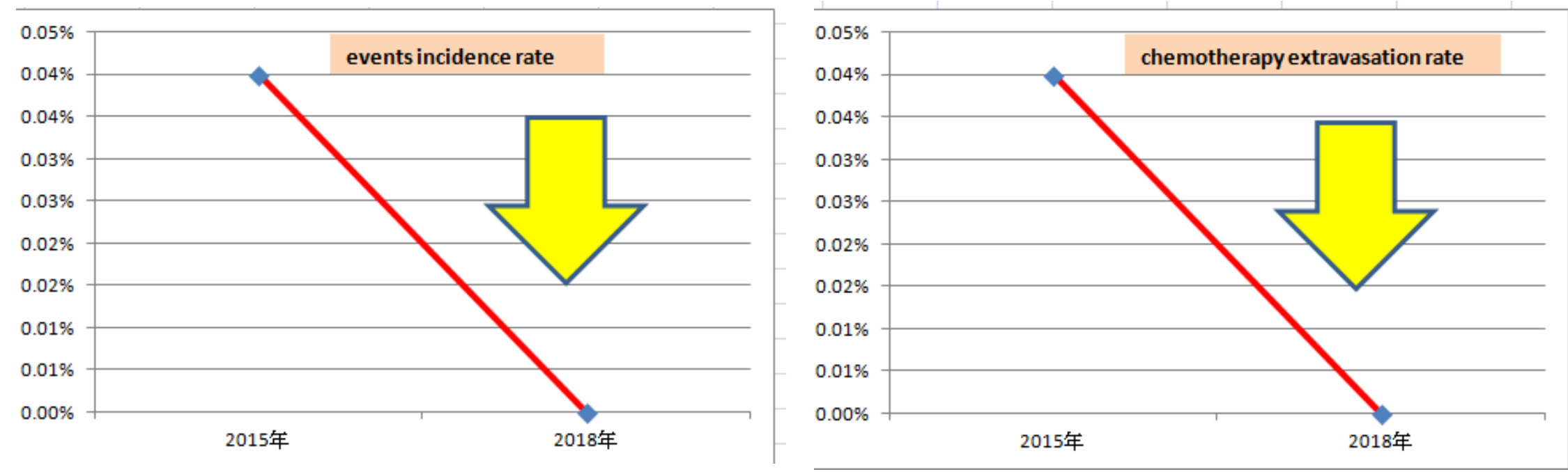


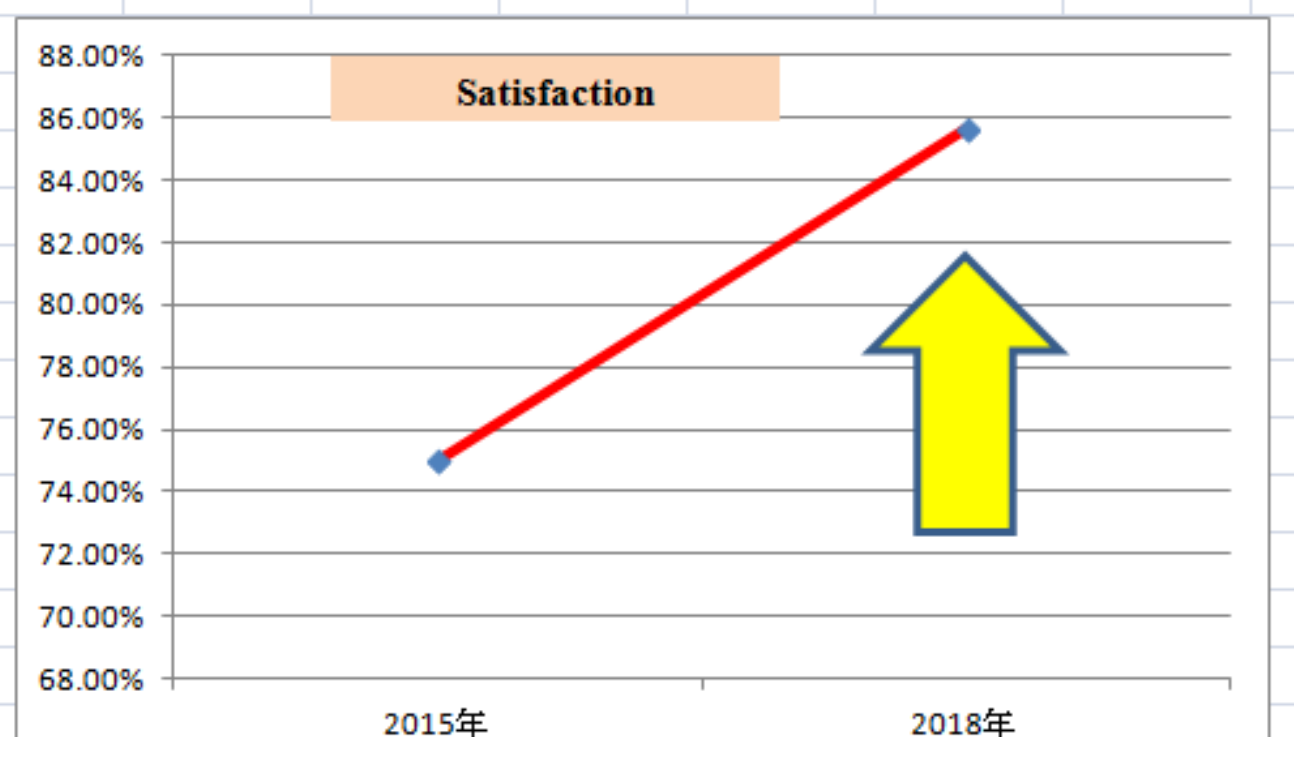




\section{RESULTS}

The chemotherapy administration events incidence rate reduced from $0.04 \%$ to $0 \%$; chemotherapy extravasation rate reduced from $0.04 \%$ to $0 \%$; the chemotherapy administration time was also shortened by 120 seconds each. Satisfaction of the medication barcode administration system also rose from 75.0 points to 85.6 points from January 2015 to September 2018. 


\section{CONCLUSION}

Team resource management was central to this project. It not only enhanced professional competence, but also improved the chemotherapy administration safety. 
Thanks for Your Attention! 